\section{ORIGINAL RESEARCH}

W.P. Daugherty

A. Ehteshami Rad

J.B. White

P.M. Meyers

G.L. Lanzino

H.J. Cloft

J. Gordon

D.F. Kallmes

\title{
Observer Agreement Regarding the Necessity of Retreatment of Previously Coiled Recurrent Cerebral Aneurysms
}

BACKGROUND AND PURPOSE: The decision regarding whether or not to retreat a previously treated aneurysm not only directly impacts patient care but also serves as a primary outcome measure in numerous, leading randomized controlled trials of modified coils. Our aim was to determine the degree of interobserver variability regarding the need and type of treatment for recurrent aneurysms following coil embolization.

MATERIALS AND METHODS: Twenty-seven previously treated recurrent aneurysms were identified. Five independent readers rated each aneurysm on a 5-point scale: 1 , definitely do not retreat; 2 , probably do not retreat; 3 , unsure; 4 , probably retreat; and 5 , definitely retreat. The readers noted, in grades $2-5$, the type of retreatment preferred, including simple coiling, balloon- or stent-assist coiling, or surgical clipping. Intraobserver agreement $\kappa$ was calculated. Retreatment recommendations were evaluated between observers by using a Wilcoxon signed rank comparison. Descriptive statistics were performed for categoric treatment-type comparisons.

RESULTS: At least 2- or 3-point differences between 2 readers were present in 17 (63\%) and 11 (41\%) of 27 cases, respectively. The median $\kappa$ was 0.27 (range, 0.04-0.43), which indicates fair agreement. Differences between readers varied, with readers 4 and 5 more often recommending retreatment compared with reviewers $1-3(P<.05)$. Wide variation was noted in treatment approach, with recommendations for surgical clipping ranging from $2(7 \%)$ to $18(67 \%)$ of 27 cases between readers 1 and 5.

CoNCLUSIONS: The current study demonstrates substantial variability among observers not only in whether to retreat a recurrent aneurysm but also how to treat it. These findings suggest that patient management varies widely across treating physicians and also calls into question the use of "retreatment" as an objective end point in clinical trials.

ABBREVIATIONS: DSA = digital subtraction angiography; $\mathrm{SAH}=$ subarachnoid hemorrhage; $\mathrm{NCRR}=$ National Center for Research Resources; $\mathrm{NIH}=$ National Institutes of Health; Post $=$ after; Pre $=$ before

$\mathbf{T}^{\mathrm{s}}$ he use of endovascular embolization of cerebral aneurysms is widespread and has become the standard of care for treatment of many intracranial aneurysms, ${ }^{1-5}$ based on favorable data from large randomized clinical trials. ${ }^{2,6}$ Following endovascular therapy, concern for recurrence and the decision to retreat aneurysms have important ramifications. ${ }^{7}$ Patients must return for ongoing follow-up, with resultant anxiety and

Received March 22, 2010; accepted after revision August 20.

From the Departments of Neurosurgery (W.P.D., G.L.L., H.J.C., D.F.K.) and Radiology (A.E.R., G.L.L., H.J.C., D.F.K.), Mayo Clinic, Rochester, Minnesota; Department of Neuroscience and Experimental Therapeutics and the Department of Surgery (J.B.W.), Texas A\&M, College Station, Texas; Departments of Radiology and Neurological Surgery (P.M.M.), Columbia University, New York, New York; and Consulting Radiologists, Ltd (J.G.), Minneapolis, Minnesota.

Statistical analysis was supported by grant number 1 UL1 RR024150 from the NCRR, a component of the NIH, and the NIH Roadmap for Medical Research. Information on NCRR is available at http://www.ncrr.nih.gov/. Information on Reengineering the Clinical Research Enterprise can be obtained from http://nihroadmap.nih.gov.

The contents of this article are solely the responsibility of the authors and do not necessarily represent the official view of the NCRR or NIH.

Please address correspondence to David F. Kallmes, MD, Mayo Clinic, Department of Radiology, 200 First St SW, Rochester, MN 55905; e-mail: kallmes.david@mayo.edu

Indicates open access to non-subscribers at www.ajnr.org

DOI 10.3174/ajnr.A2336 expense. Additionally, retreatment procedures, either with open surgery or via endovascular means, are not without risk. ${ }^{8}$ Furthermore, the "need to retreat" is a primary outcome in many previous case series as well as ongoing randomized clinical trials comparing coil types. ${ }^{9}$

Our understanding of the natural history of aneurysm remnants and recurrences continues to evolve. Even though aneurysm retreatment is entrenched as a widely accepted routine procedure for recurrent aneurysms, ${ }^{8,10,11}$ the angiographic criteria that define a "need to retreat" have received little or no attention in the literature. ${ }^{12}$ As best we can tell, the ability to place additional coils into a recurrent aneurysm cavity likely is 1 criterion, especially for endovascular retreatment considerations. ${ }^{3-5,13}$ However, we are unaware of any specific size or morphology criterion that indicates that an aneurysm should be retreated. The use of adjunctive techniques such as balloon remodeling or stent-assisted coiling probably impacts the definition of "need to retreat," especially in centers not prone to open neurosurgical re-intervention.

Given the clinical and research relevance of aneurysm-retreatment categorization, it should be reproducible across multiple practitioners or researchers. To our knowledge, there exists no formal study of the interobserver variability associated with retreatment following coil embolization. In the current study, we determined the degree of interobserver variabil- 


\section{Case 9}

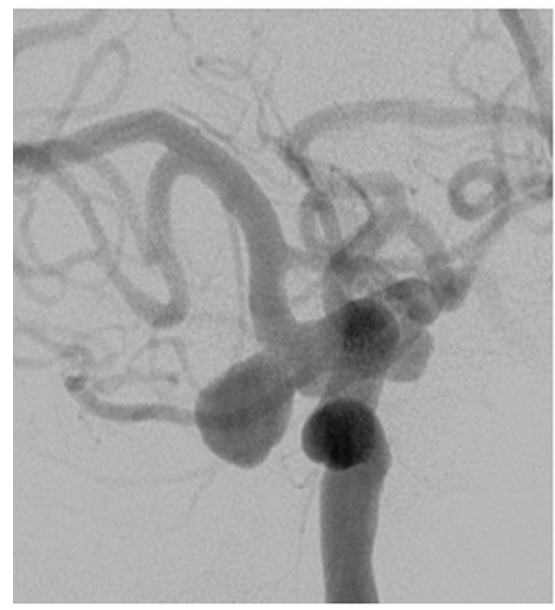

PRE

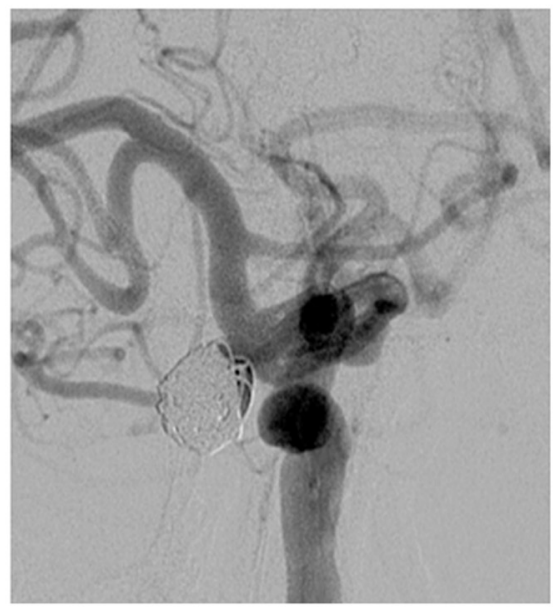

POST

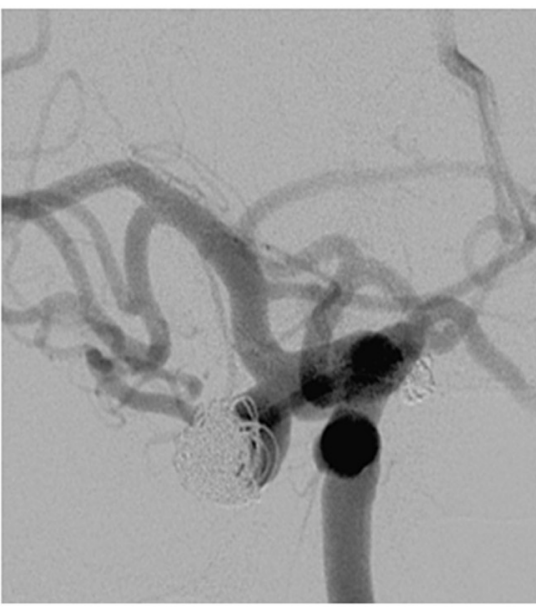

FOLLOW-UP

Fig 1. DSA in the anteroposterior plane showing the initial aneurysm conformation (Pre), the initial postcoiling final angiogram (Post), and the follow-up angiogram (Follow-Up) demonstrating recurrence. Case 9 of the series demonstrates a carotid terminus aneurysm for which all the reviewers were in complete agreement. Each reviewer scored this aneurysm as a grade 5 , definitely retreat.

\section{Case 21}

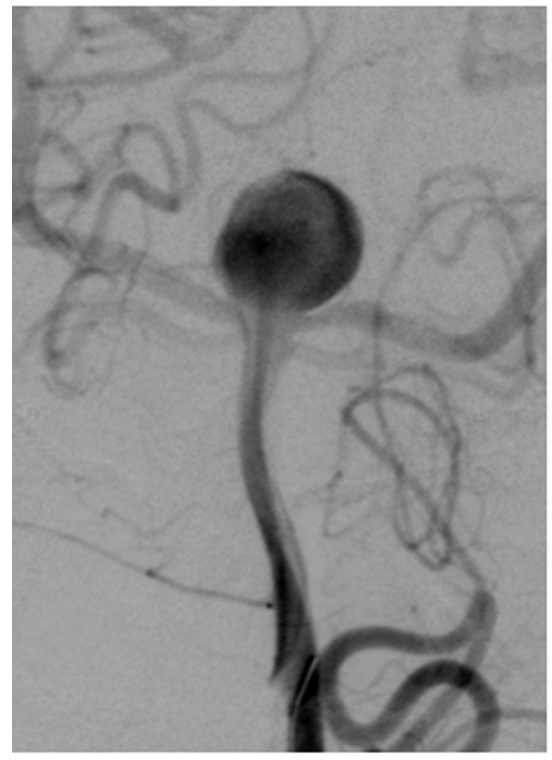

PRE

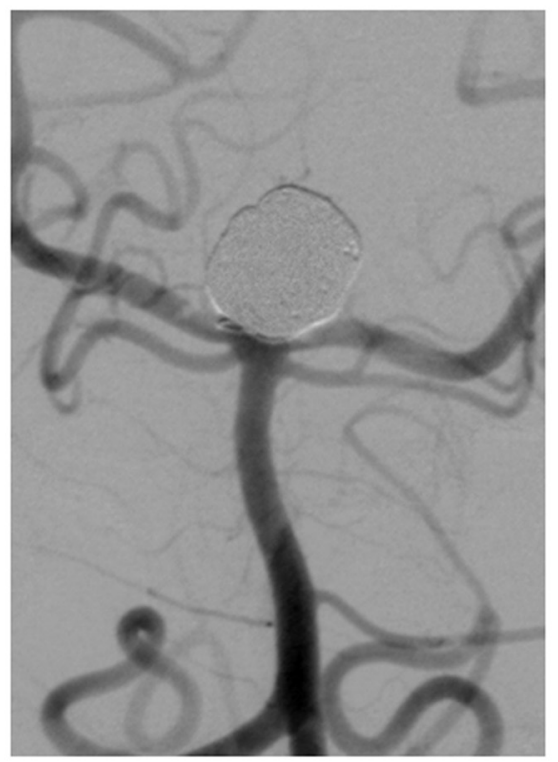

POST

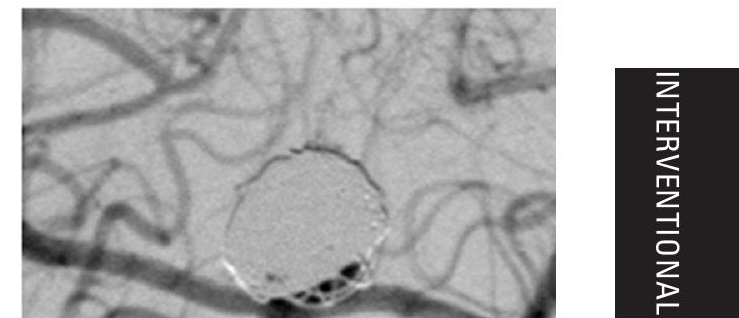

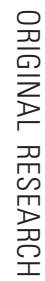

Fig 2. DSA in the anteroposterior plane showing the initial aneurysm conformation (Pre), the initial postcoiling final angiogram (Post), and the follow-up angiogram (Follow-Up) demonstrating recurrence. Case 21 demonstrates a large basilar caput aneurysm that had a complete occlusion with the initial treatment and a recurrence with recanalization of the base. The average score for this aneurysm was a $2.6 \pm 1.8$, with scores of $1,1,5,4$, and 2 . This case represents the highest variability of the 27 rated cases.

ity regarding the need and type of treatment required for recurrent or residual aneurysms following coil embolization.

\section{Materials and Methods}

Following institutional review board approval, 27 cases of previously coiled aneurysms with documented recurrence were compiled. Most had been previously identified in a prospective trial of contrast-enhanced MR angiography in the follow-up of coiled intra- cranial aneurysms, ${ }^{14}$ and the remainder were supplemented by recurrences identified during routine follow-up of previously coiled aneurysms. Aneurysm recurrence was the criterion for inclusion in the study series to prevent morphologic bias. These images were then compiled, de-identified, and placed into a 27-page PDF file (Adobe Systems, San Jose, California). Each page included a DSA of the initial uncoiled aneurysm if available, the final postcoiling workingprojection DSA, and the follow-up DSA demonstrating the recur- 
rence. The file was then distributed to 5 practitioners, including 2 endovascular neurosurgeons with 3 and 7 years' experience, respectively, and 3 interventional neuroradiologists, 2 with 13 years' experience and 1 with 12 years' experience. Two of the neuroradiologists were trained in the same fellowship and have been in practice together for 7 years, and 1 of the neurosurgeons was a fellow with these 2 neuroradiologists.

For the purposes of the current study, to diminish heterogeneity among responses based on clinical rather than imaging findings, we told the reviewers to make the following assumptions for all $27 \mathrm{pa}-$ tients: 1) The patient was a 50-year-old women who had an SAH from the index aneurysm, 2) she made a full clinical recovery, and 3) her follow-up was at 6 months.

These same observers independently reviewed each set of images and graded the need for retreatment, if any, for each case. We used a 5-point graded response in which grade 1 was defined as "definitely do not retreat," grade 2 was "probably do not retreat," grade 3 was "unsure regarding need for retreatment," grade 4 was "probably should retreat," and grade 5 was "definitely should retreat." The reviewer was then asked to specify the exact mode of retreatment, if retreatment was to be undertaken. Modes of retreatment included coil embolization without adjunct, coil embolization with balloon assist, coil embolization with stent assist, or surgical clipping of the recurrent or residual aneurysm.

\section{Statistical Analysis}

All statistics were performed by using JMP 7.0.1 software (SAS Institute, Cary, North Carolina). $\kappa$ scores were calculated between reviewers and are reported as the median and range. Plots were made for individual reviewer treatment ratings, and analysis was made between pairs of reviewers on the recommendation to retreat, by using a Wilcoxon signed rank test. Additional evaluation of adjunctive measures used to treat the recurrence was analyzed by using a Bowker test.

\section{Results}

\section{Interobserver Variability for Retreatment}

Representative cases are shown in Figs 1 and 2. The calculated agreement $\kappa$ of the 5 observers over the 27 cases presented was $\kappa=0.27$ (range, $0.04-0.43$ ) with a $\kappa$ of 0.0 being equal to random chance and 1.0 being perfect agreement. A 3- or 4-point difference between at least 2 readers was present in 10 $(37 \%)$ and $2(7 \%)$ cases, respectively. Twenty-three $(85 \%)$ of 27 cases showed at least a 1-point difference between at least 2 readers, while 17 (63\%) of the 27 cases had at least a 2-point difference between at least 2 readers. Figure 3 demonstrates the distribution of each reviewer's responses on the 5-point scale. Analysis between reviewers demonstrates that reviewers 4 and 5 were more likely to recommend retreatment than reviewers $1-3(P<.05)$. When comparing recommendations to retreat, we found no difference based on the reviewers' training (ie, neurosurgery versus neuroradiology) in the current series.

\section{Recommended Retreatment Technique}

The adjunct recommended for retreatment varied, depending on the strength of the retreatment. For cases that were scored a 2 or 3 (probably do not retreat and unsure), surgical clipping was most commonly recommended (53\% of 2 s and $83 \%$ of $3 \mathrm{~s}$ ). When retreatment recommendations were a 4 or 5 (prob-

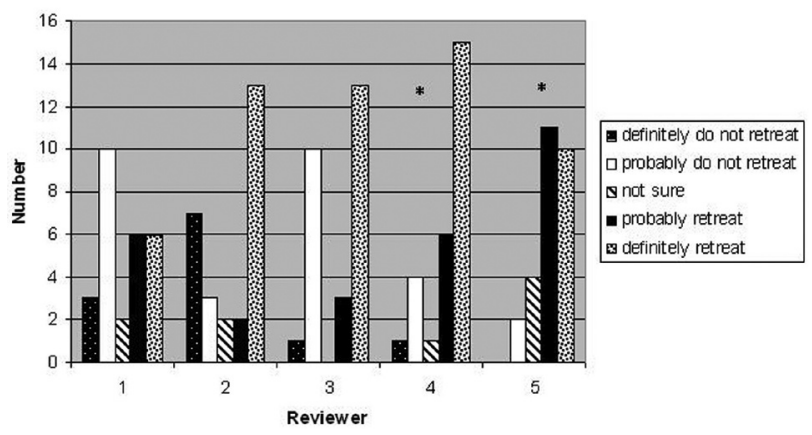

Fig 3. Relative tendency to recommend retreatment across readers. Readers responses on the need to retreat by using the 5 -point retreatment scale. Readers 4 and 5 were more likely to recommend retreatment than observation compared with readers 1-3 (asterisk indicates $P<.05)$

\section{Treatment Adjuncts}

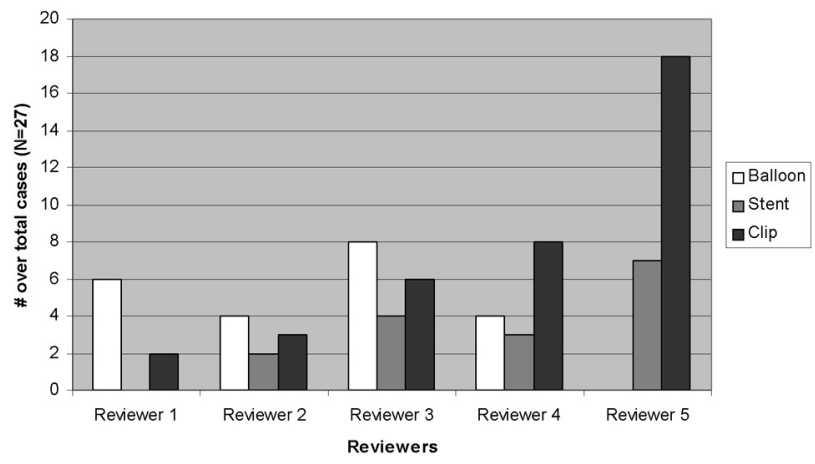

Fig 4. Treatment adjunct per reviewer. Plot of the recommended adjunctive treatment technique for each of the 5 reviewers by the total number of cases. No significant difference between reviewers and adjuncts was found by using the Bowker test for significance. There was a trend toward significance for readers 1 and $5(P<.07)$.

ably retreat and definitely retreat), surgical clipping was recommended in $29 \%$ and $28 \%$ of cases, respectively, whereas primary coiling was recommended in $25 \%$ and $39 \%$, respectively. Stent placement in this group was recommended in $25 \%$ and $7 \%$, respectively. Because retreatment was more strongly recommended, primary coiling was more often recommended over surgical clipping or stent placement. No difference was found when comparing the retreatment technique on the basis of reviewers' training (neurosurgery versus neuroradiology).

\section{Discussion}

In this study, we demonstrated substantial variability among experienced operators when deciding, on morphologic features alone, whether a series of aneurysms should be retreated and how. This finding is important for several reasons. First, it suggests that patient management following aneurysm recurrence may vary widely among operators and centers. Second, these findings suggest that retreatment may not be an ideal outcome in a clinical trial, given wide interobserver variability. Third, it sheds light on a previously neglected aspect of patient care-that is, whom should we retreat and why?

The underlying reasons behind the wide variation among our readers remain unknown but likely are multifactorial. Is- 
sues such as ease of retreatment probably impacted individual ratings - that is, rounded narrow-necked recurrences, irrespective of size, might have prompted some reviewers to recommend treatment. Difficult wide-necked cases might have prompted those who favor stents to recommend retreatment. This tendency is shown by the fact that in cases in which readers were uncertain about retreatment, surgery was recommended, while in cases with high degrees of certainty, readers were amenable to coil embolization without the need for a stent.

In our study, roughly two-thirds of cases showed differences of $\geq 2$ points. A 2 -point difference indicates a difference of "not sure" to "definitely treat" or "definitely do not treat", or a difference of "probably treat" to "probably not treat." Thus, even a 2-point difference might lead to differences in patient management. Thirty-seven percent of cases varied by 3 points. A 3-point variation indicates a difference between "definitely treat" to "probably not treat" or vice versa, again indicating substantial differences in patient management.

One might theorize that treating physicians may be more likely to recommend retreatment for ruptured recurrent aneurysms over unruptured recurrences in certain cases. To prevent this possible bias in the current study, we told the reviewers to assume that all patients were 50-year-old women who had SAHs from the index aneurysm and who had made full clinical recoveries, and that their follow-up imaging was performed at their 6-month return visit. This information was included to prevent retreatment bias based on rupture status, age, and recovery status; however, these likely play a strong role in a provider's decision about recurrent aneurysm retreatment. More research is needed in this area. Likewise, we cannot be certain that our small cohort is typical of all recurrent aneurysms. It remains possible that these cases were particularly challenging. Future studies are needed to address this issue.

Several studies have been performed evaluating the occlusion rates of aneurysms and their recurrence. ${ }^{13,15-19}$ Previous evaluations of intraobserver agreement in the assessment of aneurysm recurrence have found that agreement among observers expectedly improved as the number of response options decreased. ${ }^{12}$ Likewise studies have demonstrated that recurrence depends on the initial size of the aneurysm as well as previous rupture status. ${ }^{13,17}$ A large meta-analysis by Ferns et $\mathrm{al}^{20}$ suggested that approximately $20 \%$ of all coiled aneurysms recur and half of these go on to be retreated, but there are no good guidelines that can help a clinician decide whether observation or retreatment is more appropriate. Much of our decision-making about retreatment of recurrent aneurysms relies on experience and is likely affected by previous cases and poor outcomes.

This study has several limitations. First, 4 of the readers had overlapping training often with similar training styles. Nevertheless, the reviewer most likely to recommend observation and the reviewer most likely to recommend retreatment worked together. Second, previous studies from our group ${ }^{12}$ have suggested, as is evident in our current data, that the increase in number of rating options leads to a decrease in the intraobserver agreement $(\kappa=0.27)$ as seen in our data. Likewise, the current study was not designed to look at which part of aneurysm morphology is more likely to evoke a response advocating retreatment. Additional studies are needed to further elucidate morphometric differences that would aid in defining the need for retreatment.

\section{References}

1. Molyneux A, Kerr R, Stratton I, et al. International Subarachnoid Aneurysm Trial (ISAT) of neurosurgical clipping versus endovascular coiling in 2143 patients with ruptured intracranial aneurysms: a randomised trial. Lancet 2002;360:1267-74

2. Molyneux AJ, Kerr RS, Yu LM, et al. International Subarachnoid Aneurysm Trial (ISAT) of neurosurgical clipping versus endovascular coiling in 2143 patients with ruptured intracranial aneurysms: a randomised comparison of effects on survival, dependency, seizures, rebleeding, subgroups, and aneurysm occlusion. Lancet 2005;366:809-17

3. Raymond J, Roy D, Bojanowski M, et al. Endovascular treatment of acutely ruptured and unruptured aneurysms of the basilar bifurcation. J Neurosurg 1997;86:211-19

4. Roy D, Raymond J, Bouthillier A, et al. Endovascular treatment of ophthalmic segment aneurysms with Guglielmi detachable coils. AJNR Am J Neuroradiol 1997;18:1207-15

5. Raymond J, Guilbert F, Weill A, et al. Long-term angiographic recurrences after selective endovascular treatment of aneurysms with detachable coils. Stroke 2003;34:1398-403

6. Ryttlefors M, Enblad P, Kerr RS, et al. International subarachnoid aneurysm trial of neurosurgical clipping versus endovascular coiling: subgroup analysis of 278 elderly patients. Stroke 2008;39:2720-26

7. Kaufmann TJ, Huston J, 3rd, Mandrekar JN, et al. Complications of diagnostic cerebral angiography: evaluation of 19,826 consecutive patients. Radiology 2007;243:812-19

8. Slob MJ, Sluzewski M, van Rooij WJ, et al. Additional coiling of previously coiled cerebral aneurysms: clinical and angiographic results. AJNR Am J Neuroradiol 2004;25:1373-76

9. Kallmes DF, Cloft HJ. Ready or not, here they come: randomized trials evaluating new endovascular aneurysm therapies. AJNR Am J Neuroradiol 2007;28:799-803

10. Boet R, Poon WS, Yu SC. The management of residual and recurrent intracranial aneurysms after previous endovascular or surgical treatment: a report of eighteen cases. Acta Neurochir (Wien) 2001;143:1093-101

11. Renowden SA, Koumellis P, Benes V, et al. Retreatment of previously embolized cerebral aneurysms: the risk of further coil embolization does not negate the advantage of the initial embolization. AJNR Am J Neuroradiol 2008;29:1401-04

12. Cloft HJ, Kaufmann T, Kallmes DF. Observer agreement in the assessment of endovascular aneurysm therapy and aneurysm recurrence. AJNR Am J Neuroradiol 2007;28:497-500

13. Murayama Y, Nien YL, Duckwiler G, et al. Guglielmi detachable coil embolization of cerebral aneurysms: 11 years' experience. J Neurosurg 2003;98:959-66

14. Kaufmann TJ, Huston JI, Cloft HJ, et al. A prospective trial of $3 \mathrm{~T}$ and 1.5T time-of-flight and contrast-enhanced MR angiography in the follow-up of coiled intracranial aneurysms. AJNR Am J Neuroradiol 2010;31:912-18

15. Piepgras DG, Brown RD Jr. Management of unruptured intracranial aneurysms: perspectives on endosaccular coiling and persistent uncertainties. Stroke 2008;39:743-44

16. Gruber A, Killer M, Bavinzski G, et al. Clinical and angiographic results of endosaccular coiling treatment of giant and very large intracranial aneurysms: a 7-year, single-center experience. Neurosurgery 1999;45:793-803, discussion 803-04

17. Cognard C, Weill A, Spelle L, et al. Long-term angiographic follow-up of 169 intracranial berry aneurysms occluded with detachable coils. Radiology 1999;212:348-56

18. Kawanabe Y, Sadato A, Taki W, et al. Endovascular occlusion of intracranial aneurysms with Guglielmi detachable coils: correlation between coil packing density and coil compaction. Acta Neurochir 2001;143:451-55

19. Sluzewski M, van Rooij WJ, Slob MJ, et al. Relation between aneurysm volume, packing, and compaction in $\mathbf{1 4 5}$ cerebral aneurysms treated with coils. Radiology 2004;231:653-58

20. Ferns SP, Sprengers ME, van Rooij WJ, et al. Coiling of intracranial aneurysms: a systematic review on initial occlusion and reopening and retreatment rates. Stroke 2009;40:e523-29 